\title{
PREVALÊNCIA DE ALTERAÇÕES DE FALA EM CRIANÇAS POR MEIO DE TESTE DE RASTREAMENTO
}

\section{Prevalence of speech disorders in children through screening test}

\author{
Helena Jesini Meira Caldeira ${ }^{(1)}$, Stéffany Lara Nunes Oliveira Antunes ${ }^{(2)}$, \\ Luiza Augusta Rosa Rossi-Barbosa (3), Daniel Antunes Freitas (4), \\ Mirna Rossi Barbosa ${ }^{(5)}$, Antônio Prates Caldeira ${ }^{(6)}$
}

\begin{abstract}
RESUMO
Objetivo: conhecer a prevalência de alterações de fala em crianças que frequentam o $1^{\circ}$ ano do ensino fundamental em escolas públicas de Montes Claros - MG no ano de 2010, por meio de teste de rastreamento, verificar os resultados da triagem com a variável sexo e conhecer os principais processos fonológicos presentes nas crianças pesquisadas. Método: utilizou-se o Teste de Rastreamento de Distúrbios Articulatórios de Fala - TERDAF adaptado, um instrumento validado para este fim. Os alunos foram selecionados aleatoriamente para a aplicação do teste. Resultados: foram avaliadas 404 crianças com média de idade de seis anos e cinco meses, 52,0\% eram do sexo feminino. A prevalência de alterações de fala foi de $33,7 \%$. Pode-se observar presença de ceceio em $8,4 \%$ das crianças. Dos 137 alunos com alguma alteração nos processos fonológicos, os mais utilizados foram: substituição de /N/ por /i/ ou /y/, /// por /r/, /z/ por /s/ e omissão do /// e /r/. A chance de alterações de fala em crianças do sexo masculino foi 2,53 vezes àquela do sexo feminino. A média do tempo gasto durante o teste foi de um minuto e dezoito segundos. Conclusão: a prevalência de crianças com alterações de fala está em acordo com outros estudos. Ações do profissional fonoaudiólogo em pareceria com educadores contribuem para facilitar a aprendizagem como medida preventiva.
\end{abstract}

DESCRITORES: Prevalência; Transtornos da Articulação; Programas de Rastreamento; Fonoaudiologia

\section{INTRODUÇÃO}

A comunicação verbal é de extrema importância, seja nas relações interpessoais, seja como meio de aprendizagem. Antes que possam ter qualquer compreensão da leitura e escrita, as crianças devem entender que a fala é composta por unidades sonoras mínimas, os fonemas, e que esses podem ser representados ${ }^{1}$.

(1) Fonoaudióloga graduada pelas Faculdades Unidas do Norte de Minas - FUNORTE, Montes Claros, MG, Brasil.

(2) Fonoaudióloga graduada pelas Faculdades Unidas do Norte de Minas - FUNORTE, Montes Claros, MG, Brasil.

(3) Fonoaudióloga; Professora Mestre do Curso de Fonoaudiologia das Faculdades Unidas do Norte de Minas FUNORTE, Montes Claros, MG, Brasil.

(4) Odontólogo; Professor dos Cursos de Fonoaudiologia e Odontologia das Faculdades Unidas do Norte de Minas - FUNORTE, Montes Claros, MG, Brasil; Mestre em
Alterações na fala podem ser encontradas tais como: substituições, omissões e/ou distorções dos sons, podendo estar relacionadas às dificuldades da língua (o que caracterizaria uma dificuldade cognitivo-linguística), com a percepção auditiva dos sons e/ou com a produção dos mesmos².

O desvio fonético tem como principais causas as alterações de estruturas ósseas e/ou musculares, envolvidas na articulação e nas alterações de

Odontologia pela Universidade Vale do Rio Verde - UNINCOR, Três corações, MG, Brasil.

(5) Fonoaudiolóloga; Mestranda em Ciências da Saúde da Universidade Estadual de Montes Claros - UNIMONTES, Montes Claros, MG, Brasil.

(6) Médico pediatra; Professor Doutor dos Cursos de Mestrado e Doutorado em Ciências da Saúde da Universidade Estadual de Montes Claros - UNIMONTES, Montes Claros, MG, Brasil.

Conflito de interesses: inexistente 
produção da fala. O ceceio é um exemplo desse desvio em decorrência de uma alteração na postura da língua sendo pronunciado com apoio da língua anteriorizada, entre os dentes, ou posteriorizada, ocorrendo principalmente na produção dos fonemas $\mathrm{s} / \mathrm{e} / \mathrm{z} / 3$.

O desvio fonológico, por sua vez, ocorre na ausência de alterações orgânicas originando-se nas dificuldades de contextualização dos sons, na organização linguística e na combinação dos traços fonêmicos ${ }^{4}$.

O processo de aquisição e desenvolvimento fonológico ocorre de maneira gradual, até que haja o estabelecimento do sistema fonológico, de acordo com a comunidade linguística que a criança está inserida ${ }^{5}$. Geralmente acontece por volta dos cinco anos, podendo estender-se dos quatro até no máximo seis anos ${ }^{6}$.

Os Processos Fonológicos ocorrem quando a criança produz a fala dos adultos de forma mais fácil para ela, provocando simplificações que afetam classes de sons e não sons específicos ${ }^{7,8}$.

O interesse de se pesquisar a prevalência das alterações de fala em relação aos processos fonológicos surgiu a partir do Programa Saúde na Escola - PSE que tem como finalidade contribuir para a formação integral dos estudantes da rede pública de educação básica por meio de ações de prevenção, promoção e atenção à saúde ${ }^{9}$.

As ações em saúde previstas no âmbito do PSE deverão ser desenvolvidas articuladamente com a rede de educação pública básica e em conformidade com os princípios e diretrizes do Sistema Único de Saúde - SUS. Cabe ao fonoaudiólogo desenvolver ações, em parceria com os educadores, que contribuam para a promoção, aprimoramento, e prevenção de alterações dos aspectos relacionados à audição, linguagem (oral e escrita), motricidade oral e voz e que favoreçam e otimizem o processo de ensino e aprendizagem ${ }^{10}$.

Para verificar problemas de fala numa população se faz necessário um teste de triagem e o Teste de Rastreamento de Distúrbios de Fala - TERDAF foi validado para este fim, mas houve recomendações de ajustes para estudos posteriores ${ }^{11}$. Pesquisa com novas adaptações ao teste foram propostas, como inserção de contagem de um a dez para melhor observação do ceceio e substituições de algumas figuras ${ }^{12}$.

Os objetivos deste estudo foram: conhecer a prevalência das alterações de fala em crianças que frequentam $01^{\circ}$ ano do ensino fundamental em escolas públicas de Montes Claros - MG no ano de 2010, por meio do TERDAF adaptado ${ }^{12}$; verificar os resultados da triagem com a variável sexo e conhecer os principais processos fonológicos alterados nas crianças pesquisadas.

\section{MÉTODO}

Este trabalho caracteriza-se por um estudo transversal, analítico, realizado na cidade de Montes Claros - MG. A população alvo foi formada por crianças da $1^{\text {a }}$ série do ensino fundamental, regularmente matriculadas em escolas públicas da zona urbana de Montes Claros, no ano de 2010, subordinadas à Secretaria de Estado da Educação de Minas Gerais.

Empregou-se a técnica de amostragem aleatória por conglomerado em dois estágios, primeiro foram sorteadas as escolas e posteriormente a turma da escola sorteada. Participaram 23 escolas, sendo 16 estaduais e 7 municipais. A amostra constituiu de crianças, de ambos os sexos, cujos pais autorizaram a participação das mesmas na pesquisa e assinaram o Termo de Consentimento Livre e Esclarecido. Foram autorizados 404 termos para a aplicação do TERDAF adaptado ${ }^{12}$.

As crianças foram submetidas ao teste individualmente, na biblioteca da escola ou em uma sala vazia com pouco ruído e aplicado por duas acadêmicas do curso de Fonoaudiologia do $7^{\circ}$ período que haviam sido, previamente, treinadas e calibradas por uma profissional com experiência na área.

O instrumento utilizado, (TERDAF adaptado) ${ }^{12}$ contém vinte figuras com os fones do português brasileiro (Figura 1). No processo de readaptação do teste, a figura da borboleta foi substituída pela gravura de uma porta; a figura da placa, pela bicicleta; a figura da zebra pela gravura de uma cobra; e uma figura melhor representativa para a emissão da palavra caminhão, conforme sugestão de estudo prévio $^{12}$. Foi mantida a contagem de 1 a 10 para facilitar a avaliação do ceceio, ou seja, a produção dos fonemas /s/ e /z/ com apoio da ponta da língua entre os dentes. É importante ressaltar que foi cronometrado o tempo de aplicação da triagem.

O protocolo classifica como "normal" quando todas as respostas são corretas; "alterado" quando uma ou mais respostas são emitidas com alguma alteração no que refere aos processos fonológicos; e "inconclusivo" quando a criança deixa de falar o nome de alguma figura, por não conhecê-la ou substituí-la por outro nome, impossibilitando a avaliação do fonema em questão. As produções foram transcritas para posterior análise, realizada por duas fonoaudiólogas. Por ser um teste de rastreamento, a criança com qualquer produção emitida incorretamente foi encaminhada para diagnóstico. 
TRIAGEM ADAPTADA - FOLHA 1
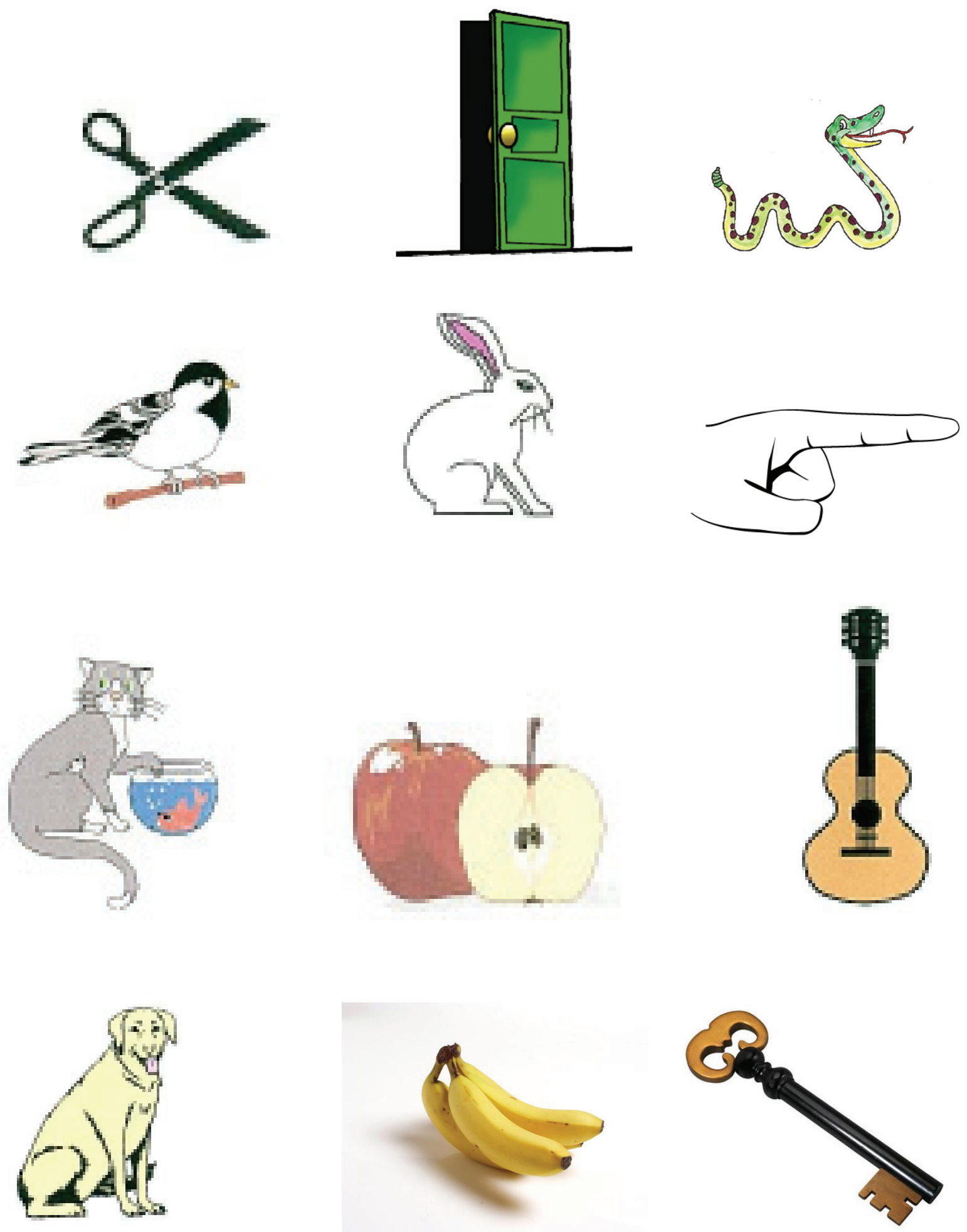
TRIAGEM ADAPTADA - FOLHA 2
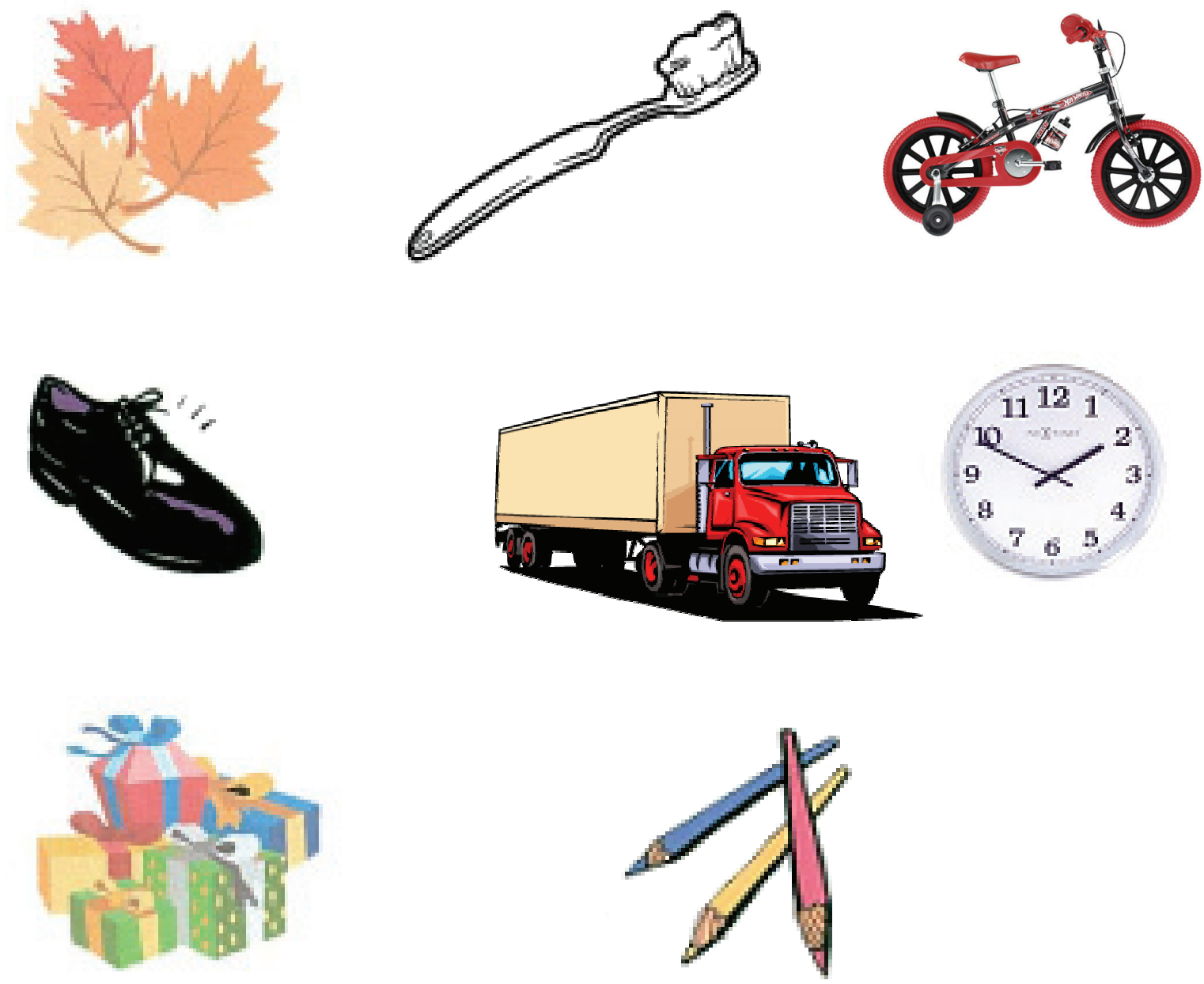

CONTAR DE 1 A 10.

Figura 1 - Etapa da pesquisa

O presente estudo foi aprovado pelo Comitê de Ética em Pesquisa da Universidade Estadual de Montes Claros - Unimontes, sob o número 1236/08.

Os dados obtidos foram analisados estatisticamente no programa Microsoft Office Excel 2007, empregado para edição de tabelas e do Software SPSS versão 17.0 para o Windows, utilizado para apreciação dos estudos estatísticos, no qual abordou a utilização do teste Qui-Quadrado $\left(\mathrm{X}^{2}\right)$ e ANOVA para observação de diferenças relevantes, assumindo-se o nível de significância de 5\% $(p<0,05)$ e foram construídos com $95 \%$ de confiança estatística.

\section{RESULTADOS}

Participaram deste estudo 404 crianças, sendo $66,3 \%(n=268)$ de escolas estaduais e $33,7 \%$ $(n=136)$, municipais; $38,6 \% \quad(n=156)$ das crianças alocadas para o estudo estudavam em escolas da área central da cidade e $61,4 \%(n=248)$ em escolas periféricas. Quanto ao sexo, 52,0\% $(n=210)$ eram meninas e 48,0\% ( $n=194)$ meninos; com idade entre cinco anos e um mês a sete anos e três meses, média de seis anos e cinco meses ( $\mathrm{DP} \pm 4,16)$. $\mathrm{Na}$ época da coleta de dados, 27 crianças $(6,7 \%)$ tinham menos de seis anos de idade. 
A média do tempo gasto durante o teste foi de um minuto e dezoito segundos. Das 404 crianças submetidas ao teste de triagem, 40,6\% $(n=164)$ apresentaram fala normal; 33,7\% $(n=136)$ apresentaram algum tipo de alteração na fala e $25,7 \%$
( $n=104)$ não reconheceram alguma das ilustrações apresentadas, sendo o teste classificado como "inconclusivo". Portanto, a prevalência de alterações de fala com teste de rastreamento foi de $33,7 \%$ (Tabela 1).

Tabela 1 - Prevalência em relação às respostas das crianças ao teste de triagem de fala; Montes Claros, MG - 2010

\begin{tabular}{ccccccccc}
\hline & \multicolumn{9}{c}{ Triagem } & \multicolumn{2}{c}{ Total } \\
\cline { 2 - 8 } Sexo & \multicolumn{2}{c}{ Normal } & \multicolumn{2}{c}{ Alterado } & \multicolumn{1}{c}{ Inconclusivo } & & \\
\cline { 2 - 8 } & $\mathbf{N}$ & $\%$ & $\mathbf{N}$ & $\%$ & $\mathbf{N}$ & $\%$ & $\mathbf{N}$ & $\%$ \\
\hline Feminino & 100 & 47,6 & 51 & 24,3 & 59 & 28,1 & 210 & 100 \\
Masculino & 64 & 33,0 & 85 & 43,8 & 45 & 23,2 & 194 & 100 \\
Total & 164 & 40,6 & 136 & 33,7 & 104 & 25,7 & 404 & 100 \\
\hline
\end{tabular}

$\mathrm{p}=0,000$

Das 136 crianças com fala alterada, $62,5 \%$ $(n=85)$ eram do sexo masculino e $37,5 \% \quad(n=51)$ do sexo feminino. Pode-se observar presença de ceceio em $8,4 \%(n=34)$ das crianças. Dentre as crianças com menos de seis anos, apenas seis delas apresentaram resultado "alterado".

Entre as 136 crianças que apresentaram emissões com alguma alteração, as mais prevalentes foram para as figuras da folha, coelho, bicicleta, presente, tesoura e cobra, sendo emitida como foia, cuêi/cuêiu, biciqueta/bicicreta, pezenti, tissora e coba respectivamente. Os processos fonológicos mais utilizados pelas crianças foram: substituição de /N/por /i/ ou /y/, /// por /r/, /z/ por /s/ e omissão do $/ / / \mathrm{e} / \mathrm{r} /$. As figuras com respostas alteradas mais recorrentes encontram-se em ordem decrescente na Tabela 2.

A tabela 3 apresenta em ordem decrescente, a frequência das figuras não reconhecidas por 104 crianças (resultado inconclusivo). No teste de rastreamento dessas crianças, a figura representativa de passarinho foi dita pela maioria como pássaro, a gravura que representa uma folha foi substituída por planta, violão foi emitido como viola, e dedo pela palavra mão.

Entre os 300 indivíduos com triagem normal e alterada foi verificada a associação entre essa variável dependente e a variável independente sexo (Tabela 4), observando-se diferença estatisticamente significante entre meninos e meninas $(p=0,000)$. O odds ratio mostrou resultado igual a 2,60 (IC 95\%: 1,63 - 4,16), portanto a chance de alterações de fala em crianças do sexo masculino é 2,6 vezes àquela em criança do sexo feminino.
Tabela 2 - Frequência de emissões realizadas pelas crianças do $1^{\circ}$ ano de escolas públicas do ensino fundamental, consideradas como alteradas na triagem de fala; Montes Claros, MG - 2010

\begin{tabular}{lcc}
\hline Figuras & $\mathbf{N}$ & $\mathbf{\%}$ \\
\hline Folha & $\mathbf{7 0}$ & $\mathbf{5 1 , 1 \%}$ \\
Coelho & $\mathbf{6 1}$ & $\mathbf{4 4 , 5 \%}$ \\
Bicicleta & $\mathbf{5 9}$ & $\mathbf{4 3 , 1 \%}$ \\
Presente & $\mathbf{4 4}$ & $\mathbf{3 2 , 1} \%$ \\
Tesoura & $\mathbf{3 9}$ & $\mathbf{2 8 , 5 \%}$ \\
Cobra & $\mathbf{2 1}$ & $\mathbf{1 5 , 3 \%}$ \\
Chave & 19 & $13,9 \%$ \\
Passarinho & 15 & $10,9 \%$ \\
Relógio & 12 & $8,7 \%$ \\
Cachorro & 9 & $6,6 \%$ \\
Porta & 9 & $6,6 \%$ \\
Gato & 8 & $5,8 \%$ \\
Violão & 8 & $5,8 \%$ \\
Sapato & 7 & $6,1 \%$ \\
Banana & 6 & $4,4 \%$ \\
Escova & 6 & $4,4 \%$ \\
Maçã & 4 & $2,9 \%$ \\
Lápis & 3 & $2,2 \%$ \\
Dedo & 1 & $0,7 \%$ \\
Caminhão & 0 & $0,0 \%$ \\
\hline
\end{tabular}

Nota: O negrito destaca as produções mais prevalentes. 
Tabela 3 - Frequência de emissões, sem reconhecimento de figura, realizadas pelas crianças do $1^{\circ}$ ano de escolas públicas do ensino fundamental; Montes Claros, MG - 2010

\begin{tabular}{lcc}
\hline \multirow{2}{*}{ Inconclusivos } & \multicolumn{2}{c}{ Respostas } \\
\cline { 2 - 3 } & No & \% \\
\hline Passarinho & 45 & $43,3 \%$ \\
Folha & 37 & $35,6 \%$ \\
Violão & 25 & $24,0 \%$ \\
Dedo & 22 & $21,1 \%$ \\
Porta & 18 & $17,3 \%$ \\
Coelho & 17 & $16,3 \%$ \\
Caminhão & 15 & $14,4 \%$ \\
Sapato & 13 & $12,5 \%$ \\
Escova & 9 & $8,6 \%$ \\
Gato & 8 & $7,7 \%$ \\
Presente & 8 & $1,7 \%$ \\
Cobra & 6 & $5,8 \%$ \\
Relógio & 5 & $4,8 \%$ \\
Maçã & 4 & $3,8 \%$ \\
Chave & 2 & $1,9 \%$ \\
Tesoura & 1 & $0,9 \%$ \\
Cachorro & 1 & $0,9 \%$ \\
Banana & 1 & $0,9 \%$ \\
Lápis & 1 & $0,9 \%$ \\
Bicicleta & 1 & $0,9 \%$ \\
\hline
\end{tabular}

A análise dos resultados, levando-se em consideração a localização das escolas (Tabela $4)$, mostrou que $51,0 \%(n=104)$ das crianças das escolas de áreas periféricas apresentaram alterações na fala, enquanto $33,3 \%(n=32)$ das crianças das escolas da região central apresentaram tais alterações. Essa diferença foi estatisticamente significante $(p=0,004)$. A substituição do $/ N /$ foi maior em crianças das escolas da periferia, em relação às crianças das escolas centrais, sendo esta diferença também estatisticamente significante (para "coelho", $p=0,032$ e para "folha", $p=0,001$ ).

Não houve diferença estatisticamente significante entre os grupos etários e o os resultados da triagem, ou seja, a proporção de crianças que apresentou alteração na triagem foi semelhante nos três grupos etários $(p=0,096)$. Entretanto, na análise da duração do tempo de realização do teste, a faixa etária se mostrou como variável associada $(p=0,000)$ (Tabela 5).

\section{DISCUSSÃO}

A prevalência de $33,7 \%$ de alterações na fala em crianças está dentro dos estudos cuja metodologia tem os mesmos critérios diagnósticos: prevalência de $26,7 \%$ foi encontrada em estudo com crianças na faixa de seis a doze anos ${ }^{11}$ e de $36,2 \%$ em estudo com crianças de cinco anos e sete meses

Tabela 4 - Associação entre variáveis estudadas e alterações de fala em crianças da primeira série de escolas públicas; Montes Claros, MG - 2010

\begin{tabular}{lcccc}
\hline Variáveis & Normal & Alterado & p-valor & OR (IC95\%) \\
\hline Sexo & & & 0,000 & \\
$\quad$ Feminino & 100 & 51 & & 1,0 \\
$\quad$ Masculino & 64 & 85 & 0,004 & $2,60(1,63-4,16)$ \\
Local das escolas & & & & 1,0 \\
$\quad$ Área central & 64 & 32 & & $2,08(1,26-3,45)$ \\
$\quad$ Área periférica & 100 & 104 & & \\
\hline
\end{tabular}

Tabela 5 - Tempo de aplicação na triagem de fala, segundo o grupo etário em crianças da primeira série de escolas públicas; Montes Claros, MG - 2010

\begin{tabular}{lccccc}
\hline Grupo etário & $\mathbf{N}$ & $\begin{array}{c}\text { Média de tempo } \\
\text { (segundos) }\end{array}$ & DP & $\begin{array}{c}\text { Teste } \\
\text { ANOVA }\end{array}$ & p-Valor \\
\hline Até 6:0 & 69 & 92,80 & 35,74 & & \\
6:1 a 6:6 & 190 & 77,24 & 31,65 & 9,604 & 0,000 \\
Acima de 6:7 & 145 & 72,80 & 29,16 & & \\
\hline
\end{tabular}


a oito anos e sete meses ${ }^{12}$. Entretanto, existem estudos na literatura brasileira que apontam prevalência de transtornos fonológicos variando de 4,2\% a $63,2 \%{ }^{13-16}$. Esta variabilidade pode ser explicada pela utilização de diferentes metodologias, idade dos sujeitos e pelo número da amostra' ${ }^{12}$. Deve-se destacar, também, que a prevalência registrada neste estudo refere-se às alterações de fala quanto aos processos fonológicos e não a transtornos fonológicos, pois não houve avaliação diagnóstica.

A maioria dos estudos indica que as alterações de fala são mais frequentes em sujeitos do sexo masculino ${ }^{4,13,17-21}$, corroborando os resultados deste trabalho. Pesquisa realizada em Salvador BA, com crianças na faixa etária de quatro a seis anos, verificou prevalência de $13,3 \%$ para o sexo masculino e $5,0 \%$ para o feminino ${ }^{13}$. Outra pesquisa realizada no Vale do Paraíba em escolares de sete anos com transtorno fonológico, $77,0 \%$ era do sexo masculino ${ }^{21}$.

Estudos realizados com pacientes atendidos em ambulatórios de Fonoaudiologia mostraram que a maior parte das crianças com transtorno fonológico era composta por meninos com idades entre cinco e sete anos ${ }^{18,19}$. Pesquisas de base populacional ou com amostras representativas de crianças em idade escolar constataram que atraso de fala, alterações fonoarticulatórias, transtorno fonológico são, aproximadamente, 1,5 vezes mais prevalentes em meninos do que em meninas ${ }^{17,20}$.

No que refere ao ceceio, este estudo tem resultados semelhantes com pesquisa realizada na mesma cidade, em escolares com média de idade de seis anos e seis meses $(\mathrm{DP} \pm 5,06)$, no qual a prevalência de desvio exclusivamente fonético foi de $10,4 \%^{12}$. Já o estudo realizado em Santa Maria - RS com crianças de idade entre cinco anos e sete meses e sete anos e cinco meses, 2,1\% apresentaram desvio fonético ${ }^{15}$. A literatura revela que estas alterações fonéticas diminui sua ocorrência com o aumento da idade ${ }^{22}$.

Observou-se, no presente estudo, diferença significante entre crianças de escolas centrais e periféricas que, possivelmente, está associada ao gradiente social. Embora, não se tenha aferido o nível socioeconômico das famílias das crianças envolvidas, a localização das escolas pode ser tomada como uma variável que, em certa medida, também é um indicador social. A substituição de /N/ por $/ y /$ foi a mais frequente no presente estudo. Tal alteração é bastante comum e caracteriza forma estigmatizada do falar urbano ${ }^{12,23,24}$. É um fenômeno linguístico antigo, desde à chegada da língua portuguesa ao Brasil, portanto, estão reproduzindo uma vocalização presente há séculos ${ }^{12}$. Há autores que descreveram que o desenvolvimento da linguagem está associado aos padrões linguísticos aos quais a criança está exposta ${ }^{25,26}$ não devendo considerar esta forma de linguagem como patológico mas sim como variação linguística ou regionalismo ${ }^{12}$.

A diferença no tempo necessário para a aplicação da triagem foi significantemente diferente entre os grupos etários. De um modo geral o tempo de aplicação diminui conforme o aumento da idade das crianças ${ }^{27} \mathrm{O}$ que pode ser explicado por fatores maturacionais e de estimulação ${ }^{28}$. A média do tempo gasto demonstrou ser um teste simples e rápido. Conforme a literatura, para o levantamento de dados em uma população relativamente grande e sem queixas deve-se utilizar um teste de rastreamento de fácil aplicação, ser rápido e validado ${ }^{29}$, aspectos que foram considerados para a realização da presente pesquisa.

Outro aspecto a ser destacado refere-se ao teste empregado. Pode-se observar que as substituições de figuras sugeridas em estudo anterior ${ }^{12}$ reduziram o percentual de resultados inconclusivos em $83,6 \%$. Quanto às demais figuras sem reconhecimento pelas crianças, podem-se adotar facilitadores. Por exemplo, na figura do passarinho: "um pássaro pequeno é um...", na figura do dedo: "na mão temos cinco...."

Os resultados deste estudo salientam a importância de medidas preventivas no processo de educação infantil e a relevância do papel do fonoaudiólogo nas escolas. Este profissional deve criar condições favoráveis e eficazes para que as capacidades de cada aluno possam ser exploradas ao máximo, não no sentido de eliminar problemas, mas baseando-se na crença de que determinadas situações e experiências podem facilitar e incrementar o desenvolvimento e a aprendizagem ${ }^{30}$.

\section{CONCLUSÃO}

A prevalência de crianças com alterações de fala está em acordo com outros estudos e também reforçam que estas ocorrem mais em crianças do sexo masculino.

Problema de fala pode ser prejudicial ao desenvolvimento da criança e sua interação no meio social. A participação do fonoaudiólogo na área educacional deve ser de orientação e planejamento escolar, criando condições para facilitar a alfabetização e etapas posteriores a ela, em medidas de caráter preventivo. 


\section{ABSTRACT}

Purpose: to determine the prevalence of speech disorders in children attending the first grade of primary education in public schools in Montes Claros - MG in 2010, through a screening test, in order to check the screening results with the sex variable and on the main phonological processes exhibited by the surveyed children. Method: we used an adopted validated instrument for this purpose (Screening Test for Speech Articulation Disorders). Results: a total of 404 children with a mean age of six years and five months and $52,0 \%$ were female. The prevalence of phonological disorders was $33,7 \%$. It was possible to observe the presence of lisp in $8.4 \%$ of children. Of the 137 students with any changes in the phonological processes, the most used were: replacement $/ N /$ with $/ \mathrm{i} /$ or $/ \mathrm{y} /, / / / \mathrm{a} / \mathrm{r} / \mathrm{,} / \mathrm{z} / \mathrm{with} / \mathrm{s} /$ and omission of $/ / /$ and $/ r /$.. The chance of phonological disorder in male children is 2,53 times that in female child. The average time spent during the test was 1 minute and 18 seconds. Conclusion: we found a high prevalence of phonological disorder demanding not only effective therapeutic intervention program, but also preventive measures. Prevalence of children with speech disorders is in agreement with other studies... Actions of the professional speech therapist in partnership with educators help to facilitate learning as a preventive measure...

KEYWORDS: Prevalence; Articulation Disorders; Screening Programs; Speech, Language and Hearing Sciences

\section{REFERÊNCIAS}

1. Papis L, Assencio-Ferreira VJ. Consciência fonológica como meio de avaliação de crianças com dificuldades de aprendizagem escolar. Rev CEFAC. 2001; 3(2):117-21.

2. Wertzner HF, Pagan LO, Galea DES, Papp ACCS. Características fonológicas de crianças com transtorno fonológico com e sem histórico de otite média. Rev Soc Bras Fonoaudiol. 2007;12(1):41-7.

3. Sociedade Brasileira de Fonoaudiologia SBFA. Comitê de Motricidade Oral (MO) [Internet]. Documento Oficial 03/2003 [cited 2005 Dez 10]. Disponível em: http://www.sbfa.org.br

4. Santos GG, Melo PDF, Diniz JMG, Teixeira GPB. A importância do diagnóstico diferencial das alterações de fala: enfoque fonológico. J Bras Fonoaudiol. 2003;4(16):186-92.

5. Mota HB, Kaminski TI, Nepomuceno MRF, Athayde ML. Alterações no vocabulário expressivo de crianças com desvio fonológico. Rev Soc Bras Fonoaudiol. 2009;14(1):41-7.

6. Vieira MG, Mota HB, Keske-Soares M. Relação entre idade, grau de severidade do desvio fonológico e consciência fonológica. Rev Soc Bras Fonoaudiol. 2004;9(3):144-50.

7. Ingram D. Aspects of phonological acquisition. In: Ingram D. Phonological disability in children. London: Edward Arnold; 1976. p.10-49.

8. Yavas MS, Hernandorena CLM. Lamprecht RR. Avaliação fonológica da criança: reeducação e terapia, Porto Alegre: Artmed. 2002. p.90-117.
9. Brasil. Decreto no 6.286, de 5 de dezembro de 2007. Institui o Programa Saúde na Escola -PSE, e dá outras providências.

10. Conselho Federal de Fonoaudiologia. Resolução CFFa no 309, de 01 de abril de 2005. Dispõe sobre a atuação do Fonoaudiólogo na educação infantil, ensino fundamental, médio, especial e superior, e dá outras providências. Brasília, 1 abr. 2005.

11. Goulart BNG, Ferreira J. Teste de rastreamento de alterações de fala para crianças - Terdaf. Pró-Fono Rev Atual Cient. 2009;21(3):231-6.

12. Rossi-Barbosa LAR. Prevalência de Transtornos Fonológicos em Crianças do 1ํㅡㄹ Ano do Ensino Fundamental [dissertação]. Montes Claros (MG): Universidade Estadual de Montes Claros UNIMONTES; 2010.

13. Cavalheiro LG. A prevalência do desvio fonológico em crianças de 4 a 6 anos de escolas públicas municipais de Salvador-BA. Rev Soc Bras Fonoaudiol. 2008;13(4):415.

14. Andrade CMF. Prevalência de desordens idiopáticas da fala e da linguagem em crianças de um a onze anos de idade. Rev. Saúde Pública. 1997; 31(5): 495-501.

15. Casarin MT. Estudos dos desvios de fala em pré-escolares de escolas públicas estaduais de Santa Maria-RS [Dissertação]. Santa Maria: Universidade Federal de Santa Maria; 2006.

16. Vitor RM, Cardoso-Martins C. Desenvolvimento fonológico de crianças pré-escolares da Região Noroeste de Belo Horizonte. Psicol rev. 2007;13(2):383-98. 
17. Shriberg LD, Tomblin JB, Mcsweeny JL. Prevalence of speech delay in 6-year-old children and comorbidity with language impairment. J Speech Lang Hear Res. 1999;42(6):1461-81.

18. Matumoto S, Mishima SM, Pinto IC. Saúde coletiva: um desafio para a enfermagem. Cad Saude Publica. 2001;17(1):233-41.

19. Wertzner HF, Oliveira MMF. Semelhanças entre os Sujeitos com Distúrbio Fonológico. Pró-Fono R Atual Cien. 2002;14(2):143-52.

20. Matida AH, Camacho LAB. Pesquisa avaliativa e epidemiologia: movimentos e síntese no processo de avaliação de programas de saúde. Cad Saude Publica. 2004;20(1):37-47.

21. Patah KL, Takiuchi N. Prevalência das Alterações Fonológicas e Uso dos Processos Fonológicos em Escolares aos 7 Anos. Rev CEFAC. 2008;10(2):158-67.

22. Pereira MMB, Bianchini EBG, Carvalho GGT, Jardim ZMG. Investigação da ocorrência e caracterização de distorções do /s/ em crianças de 3 a 10 anos. Rev Soc Bras Fonoaudiol. 2003;8(1):10-7.

23. Barrera SD, Maluf MR. Variação lingüística e alfabetização: um estudo com crianças da primeira série do ensino fundamental. Psicologia Escolar e Educacional. 2004;8(1):35-46.
24. Nery A. O modo de falar do brasileiro. Pagina 3 Pedagogia \& Comunicação. [periódico na Internet]. 2007 [acessado 2010 Ago 12]. Disponível em: http:// educacao.uol.com.br/portugues/ult1693u60.jhtm.

25. Shriberg LD, Kwiatkowski J. Developmental phonological disorders: I. A clinical profile. J Speech Hear Res. 1994;37(5):1100-26.

26. Morales MV, Mota HB, Keske-Soares M. Consciência fonológica: desempenho de crianças com e sem desvios fonológicos evolutivos. Pró-Fono Rev Atual Cient. 2002;14(2):153-64.

27. Carvalho BS. Teste de figuras para discriminação fonêmica [dissertação]. Santa Maria (RS): Universidade Federal de Santa Maria UFSM; 2007.

28. Rodrigues EJB. Discriminação auditiva normas para avaliação de crianças de 5 a 9 anos. São Paulo: Cortez; $1981 .$.

29. Lewis BA, Freebairn LA, Taylor HG. Academic outcomes in children with histories of speech sound disorders. J Commun Disor. 2000;33(1):11-30.

30. Ramos AS, Alves LM. A fonoaudiologia na relação entre escolas regulares de ensino fundamental e escolas de educação especial no processo de inclusão. Rev. Bras. Educ. Espec. 2008;14(2):235-50.
http://dx.doi.org/10.1590/S1516-18462012005000039

RECEBIDO EM: 09/05/2011

ACEITO EM: 18/10/2011

Endereço para correspondência:

Daniel Antunes Freitas

Faculdades Unidas do Norte de Minas -

FUNORTE

Avenida Osmane Brandão, s/n - Bairro JK

Montes Claros - MG

CEP: 39400-000

E-mail: danielmestradounincor@yahoo.com.br 